\title{
Implantable Brain-Computer Interface for Volitional Hand Grasp in Spinal Cord Injury
}

\section{lahn Cajigas}

University of Miami Medical School https://orcid.org/0000-0002-5808-453X

\section{Kevin Davis}

University of Miami

\section{Benyamin Meschede-Krasa}

Department of Brain and Cognitive Science

\section{Noeline Prins}

University of Ruhuna

\section{Sebastian Gallo}

University of Miami

Jasim Naeem

University of Miami

Anne Palermo

University of Miami

\section{Audrey Wilson}

University of Miami

\section{Santiago Guerra}

Department of Neurological Surgery, University of Miami Hospital

\section{Brandon Parks}

University of Florida https://orcid.org/0000-0002-1317-5358

\section{Lauren Zimmerman}

University of Miami

\section{Katie Gant}

University of Miami

\section{Allan Levi}

University of Miami, Dept. of Neurological Surgery and the Miami Project to Cure Paralysis

\section{W. Dalton Dietrich}

University of Miami

\section{Letitia Fisher}

University of Miami

\section{Steven Vanno}

University of Miami

John Tauber 
Massachusetts Institute of Technology

\section{Indie Garwood}

Massachusetts Institute of Technology

John Abel

Massachusetts Institute of Technology

\section{Emery Brown}

Massachusetts Institute of Technology

\section{Michael Ivan}

University of Miami https://orcid.org/0000-0002-4798-4989

\section{Abhishek Prasad}

University of Miami

Jonathan Jagid ( $\nabla_{\text {jjagid@med.miami.edu })}$

University of Miami

\section{Article}

Keywords: cervical spinal cord injury, hand grasp, brain-computer interface (BCl)

Posted Date: January 8th, 2021

DOl: https://doi.org/10.21203/rs.3.rs-138657/v1

License: (c) (i) This work is licensed under a Creative Commons Attribution 4.0 International License. Read Full License 


\section{Implantable Brain-Computer Interface for Volitional}

\section{Hand Grasp in Spinal Cord Injury}

3 Iahn Cajigas, MD, $\mathrm{PhD}^{1}$, Kevin C. Davis, $\mathrm{BS}^{2^{*}}$, Benyamin Meschede-Krasa, $\mathrm{MS}^{567^{*}}$,

4 Noeline W. Prins, $\mathrm{PhD}^{2,8}$, Sebastian Gallo, $\mathrm{AS}^{2}$, Jasim Ahmad Naeem, $\mathrm{MS}^{2}$, Anne Palermo,

$5 \mathrm{DPT}^{3}$, Audrey Wilson, $\mathrm{MS}^{4}$, Santiago Guerra, $\mathrm{MS}^{2}$, Brandon A. Parks, $\mathrm{BS}^{2}$, Lauren

6 Zimmerman, $\mathrm{MS}^{2}$, Katie Gant, $\mathrm{PhD}^{4}$, Allan D. Levi, MD, $\mathrm{PhD}^{1,4}$, W. Dalton Dietrich,

$7 \mathrm{PhD}^{1,2,4}$, Letitia Fisher, BA ${ }^{4}$, Steven Vanni, DO ${ }^{1,4}$, John Michael Tauber, $\mathrm{MS}^{57}$, Indie C.

8 Garwood, MSE ${ }^{57}$, John H. Abel, PhD ${ }^{567}$, Emery N. Brown, MD, PhD ${ }^{567}$, Michael E. Ivan,

$9 \mathrm{MD}, \mathrm{MBS}^{1}$, Abhishek Prasad, $\mathrm{PhD}^{2,4}$, Jonathan Jagid, $\mathrm{MD}^{1,4}$

$10{ }^{1}$ Department of Neurological Surgery, University of Miami, Miami, FL 33136

$11{ }^{2}$ Department of Biomedical Engineering, University of Miami, Miami, FL 33146

$12{ }^{3}$ Department of Physical Therapy, University of Miami, Miami, FL 33146

$13{ }^{4}$ Miami Project to Cure Paralysis, University of Miami, Miami, FL 33136

$14{ }^{5}$ Department of Brain and Cognitive Science, Massachusetts Institute of Technology, Cambridge, MA 02139

$15{ }^{6}$ Department of Anesthesia, Critical Care and Pain Medicine, Massachusetts General Hospital, Boston, MA 02114

$16{ }^{7}$ Picower Institute for Learning and Memory, Massachusetts Institute of Technology, Cambridge, MA 02139

$17{ }^{8}$ Department of Electrical and Information Engineering, University of Ruhuna, Sri Lanka

18 * Authors contributed equally

19 Corresponding author: Jonathan R. Jagid, MD

Department of Neurological Surgery

University of Miami

1095 NW 14th Terrace

Miami, FL 33136

Tel. (305) 243-3256

Fax (305) 243-6017 


\section{Abstract}

28 Loss of hand function after cervical spinal cord injury severely impairs functional independence. We describe a method for restoring volitional control of hand grasp in a subject with complete cervical quadriplegia (C5 ASIA Impairment Scale A) using a portable fully implanted brain-computer interface $(\mathrm{BCl})$ within the home environment. The $\mathrm{BCl}$ consists of subdural surface electrodes placed over the dominant-hand motor cortex and connects to a transmitter implanted subcutaneously below the clavicle, which allows continuous reading of the electrocorticographic (ECoG) activity. Movement-intent was used to trigger functional electrical stimulation (FES) of the dominant hand during an initial 29-week laboratory study and subsequently via a mechanical hand orthosis during in-home use. Movement intent information could be decoded consistently throughout the 29-week in-laboratory study with a mean accuracy of $89.0 \%$ (range $78-93.3 \%$ ). Improvements were observed in both the speed and accuracy of various upper extremity tasks, including lifting small objects and transferring objects to specific targets. After study week 23 , the subject began to be able to extend his right thumb volitionally in the absence of the FES orthosis. At home decoding accuracy during open-loop trials reached an accuracy of $91.3 \%$ (range $80-98.95 \%$ ) and an accuracy of $88.3 \%$ (range $77.6-95.5 \%$ ) during closed-loop trials. A fully implanted $\mathrm{BCl}$ can be safely used to reliably decode movement intent from motor cortex, allowing for accurate volitional control of hand grasp and may potentially re-engage latent neural pathways to allow targeted re-innervation of muscles below the level of injury. (Funded by the Miami Project to Cure Paralysis; ClinicalTrials.gov number, NCT02564419.) 
51 Spinal cord injury ( $\mathrm{SCl})$ is a devastating disease, which exerts a disproportionate medical, social, and

52 economic toll on those injured and society. Despite many exciting pre-clinical studies underway, to date,

53 no therapeutic intervention has been demonstrated to definitively improve neurological outcomes or 54 mitigate the effects of secondary neural injury. Neural interface research has been strongly motivated 55 by the need to restore the ability to communicate or improve motor function to the more than 5.4 million 56 individuals in the U.S. suffering from various neurological disorders and diseases of the central and 57 peripheral nervous system which result in paralysis such as stroke (33.7\%), SCl (27.3\%), and multiple sclerosis $(18.6 \%){ }^{1-6}$ The long-term use of rehabilitative neuroprosthetics could significantly improve the quality of life of paralyzed individuals with neurotechnology to reanimate nonfunctional limbs, replace missing limbs with neuroprosthetics, and enable new modes of direct neural communication. ${ }^{1,4}$

Over the last 20 years, there has been a surge in the number of successful applications of braincomputer interfaces (BCls) for upper extremity control involving reaching and grasping. ${ }^{7-12}$ However, fully implanted and portable motor BCls have so far not been successfully implemented and have never been deployed in an at-home setting. $\mathrm{BCl}$ systems using implanted electrodes have shown promise in controlling cursors or robotic arms, though these systems usually require subjects to be constantly tethered to an external power source and recording hardware, which limits their application to a laboratory setting. ${ }^{7,9,13}$ Additionally, $\mathrm{BCls}$ using implanted electrodes generally rely on single-neuron activity $8,10,12$, the recording quality of which is known to decline over time in animals and humans. ${ }^{14}$ Successful translation of BCls into at-home environment has been possible though these studies have generally relied on signals recorded non-invasively from the scalp via electroencephalography (EEG). ${ }^{15-}$ ${ }^{18}$ Scalp EEG recordings suffer from low signal-to-noise ratio (SNR), are prone to artifacts, and require assistance from caretakers for set-up, resulting in barriers to convenient at-home use. Other studies have instead utilized more stable electrocorticographic (ECoG) signals recorded from the brain

74 surface. ${ }^{17}$ However, until recent technological developments, these attempts have been limited to temporary implantations due to the nature of the clinical scenarios where ECoG is typically used, that 
is, seizure localization in epilepsy. Recently, two bilateral wireless epidural implants with 64 channels were shown to allow control of a four-limb neuroprosthetic exoskeleton in a laboratory setting in a subject with tetraplegia with stable decoding function over 24 months. ${ }^{19}$

In this study, we sought to evaluate the safety, efficacy, and long-term stability of a fully implanted $\mathrm{BCl}$ for control of a volitional hand grasp in a subject suffering from cervical quadriplegia via functional electrical stimulation (FES) orthosis in the acute post-operative phase within a laboratory setting and then via a motorized hand orthosis in the home environment. The final system was implemented so that

83 it could be mounted on the subject's wheelchair to provide continuous use outside the laboratory environment without the need for clinician assistance via a patient-controlled smart phone application.

\section{Methods}

\section{Screening Protocol}

All study procedures were approved by the University of Miami Institutional Review Board and the U.S. FDA (ClinicalTrials.gov: NCT02564419). A total of 21 subjects with C5/C6 motor complete spinal cord injury, according to the International Standards for Neurological Classification of Spinal Cord Injury (ISNSCI), provided written informed consent for screening with an EEG-based protocol (see Supp. Methods) to test their ability to trigger a FES device based on EEG signals produced while performing motor imagery of dominant hand movement and rest. ${ }^{20}$ Subjects had to be $18-50$ years old and have a chronic injury (greater than 1-year post-injury) with a C5 or C6 motor level according to the

94 International Standards for Neurological Classification of Spinal Cord Injury (ISNCSCI $)^{21}$ and had to 95 achieve sufficient hand opening/closing with FES to allow grasping. A total of 17 subjects participated 96 in the EEG screening over 1-10 weeks. One subject qualified for and consented to the surgical 97 implantation and completed all 16 sessions of EEG screening.

\section{Surgical Protocol}

99 Pre-operative evaluation with functional magnetic resonance imaging was used to map the site of 100 cortical activation during imagined dominant (right) hand movements and actual shoulder movements. 
Diffusion tensor imaging was used to identify the location of the corticospinal tract fibers that had previously controlled the dominant hand movement in the subject. The merged pre-operative imaging, as shown in Figure 1A, was used to plan a small craniotomy over the left motor cortex with the assistance of frameless stereotaxy (Stealth S7, Medtronic, Minneapolis, MN). Intraoperative electrical

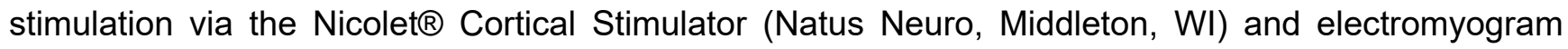
(EMG) monitoring was used to definitively identify motor cortex by evoking EMG activity in muscles proximal to the level of spinal cord injury. As determined from the pre-operative imaging and intraoperative stimulation, two four-contact electrodes (Resume II leads, Medtronic, Minneapolis, MN), were approximately centered on the hand/arm area on the left hemisphere with the long-axis of the leads oriented in the anterior-posterior direction. The leads were tunneled subcutaneously to the left subclavicular region where they were connected to the implanted transmitter (Activa PC+S, Medtronic, Minneapolis, MN). Surgical implantation occurred on November 30, 2018 with no complications and the patient was discharged home on post-operative day 2.

\section{Device}

115 The Activa PC+S (Medtronic, Minneapolis, MN) is a deep brain stimulation system that allows for realtime sensing and recording of brain activity. In this study, two-four contact electrodes were used for real-time sensing of the ECoG. The 8 contacts were configured in bipolar mode (Figure 1B) resulting in a total of four ECoG channels. Channels 1 and 3 output the real-time ECoG at a sample frequency of $200 \mathrm{~Hz}$ whereas channels 2 and 4 output the average power between $4 \mathrm{~Hz}$ and $36 \mathrm{~Hz}$ computed onboard the transmitter at sample frequency of $5 \mathrm{~Hz}$. Packets of data were transmitted every 0.4 seconds.

\section{Decoding upper extremity movement intent}

122 The subject came to the laboratory 2-3 times per week for 1-2 hours at a time. A timeline of the 29week laboratory trial is included in the supplementary methods (Figure S1). From study weeks 9-19, "closed-loop" upper extremity experiments were conducted where the decoded motor imagery state from the online classifier was used to drive FES of the right upper extremity via an external orthosis 
127 development of initial decoders are included in the supplementary methods. Figure $1 \mathrm{C}$ summarizes the 128 laboratory setup.

129 Beginning in July 2020, the continuous decoding system was deployed in the subject's home with the 130 ability to control and calibrate the system via a custom smartphone-based application. In order to allow 131 longer times of operation without the need for charging, hand grasp was actuated by a Bluetooth132 enabled battery powered mechanical hand orthosis (Neomano, Neofect, South Korea) instead of FES.

133 The orthosis, worn on the subject's dominant hand over the first three digits, drives active flexion using 134 a motor that tightens strings attached to the distal anterior end of the second and third digit. Passive 135 extension is enabled by using elastic straps attached from the distal posterior surfaces of the same digits to a Velcro surface located on the dorsum of the hand. Decoded movement states were used to send motor commands to the orthosis.

\section{At-home Decoding System}

139 The at-home system was constructed using an external battery (50000 mAh power bank, Krisdonia, 140 China), a nano computer (m90n Nano, Lenovo, China), and a custom 3D-printed casing to house the battery and computer. This casing was mounted to the back of the subject's wheelchair as shown in

Figure 1D. The computer was configured to run the $\mathrm{BCl}$ application on startup. This application was designed to load and register connected devices, including an external antenna for telemetry from the Activa PC+S and connections for the mechanical orthosis. A custom mobile phone application was designed as the user interface to read system status information, alter system settings, and initiate testing sessions by communicating with the computer over Bluetooth Low Energy (Video 2).

\section{Feature Extraction}

\section{Windowing}

149 For operation of the decoder in both laboratory and home settings, we sought to map the power spectral 150 density (PSD) of the ECoG recording to the binary move/rest state of the hand. For the continuous 151 decoder for home use, in order to track changes in the ECoG power spectrum over time, data from 
each channel were divided into overlapping windows and the PSD was computed for each window. A window size of 3.2 seconds was selected with a window step of 0.4 seconds by cross-validation (Supp. Methods). For training, only windows with non-ambiguously labeled data were used (e.g. there were no transitions between motor states within a single window). Other labelling schemes were explored (Supp. Methods).

\section{Power Spectral Estimation}

Spectral estimates from each channel were computed for each window of data and aggregated into a feature vector for motor intent decoding. For ECoG channels 1 and 3, a PSD with frequency bands ranging from $0-100 \mathrm{~Hz}$ were computed using a multitaper method ${ }^{22}$ (Supp. Methods). For channels 2 and 4, the median spectral estimate was calculated and included in the feature vector for that window.

\section{Decoding Model Architecture}

\section{In-lab decoding}

To assess the ability of ECoG PSD features to discriminate between move and rest motor states, the movement for 3 seconds. Synchronization between the displayed message (Figure 1C) and the recorded ECoG was achieved by application of a small pulse to the subject's scalp (below sensation threshold) that would cause an artifact in the recorded ECoG. The recorded data was segmented using transition pulse and labelled according to the displayed desired motor state to create dataset for classifier training. Five commonly applied machine learning classifiers were tested: bagged trees, knearest neighbors, linear discriminant, linear support vector machine, and an artificial neural network. All in laboratory classifiers were trained in Matlab 2018b, and online experiments were conducted in Matlab 2015a. Off-line classifiers were selected as outlined in Supp. Methods Table S4.

\section{At-home decoder}

175 In order to build a robust decoder used in the home, using training data with random transitions between 
switching between states. A two-step decoder architecture first used linear discriminant analysis (LDA)

In-home training data was collected in 5-minute trials where epochs of "move" (close hand) and "rest"

180 (open hand) instructions were delivered to the subject via the subject's smartphone while ECoG data

was recorded. Motor instructions were randomly chosen to last between 6 and 10 seconds (in $0.4 \mathrm{~s}$ intervals corresponding to packet transmission frequency) before transitioning to the other motor state in order to capture the random transition between motor states that would occur during real-time use.

184 Thirty-three trials performed in this manner were collected for at-home decoding algorithm training. 185 Held-out open-loop validation data was collected in the same fashion, without actuating the mechanical 186 glove, and was not used for training. Finally, held-out closed-loop validation data was collected in the same fashion, but the decoded motor state was used to control the mechanical glove online, giving the subject visual feedback of the decoder output. A detailed description of the at-home decoder architecture and other architectures that were explored in cross-validation are can be found in the Supplementary methods.

\section{Functional tasks}

From study weeks 11-19, during the in-laboratory testing, several tasks were performed alongside the upper extremity trials to quantify any improvements in upper extremity function. Starting on week 11, whenever a correct move state was decoded and the subject was receiving FES to open and close the hand, he was asked to pick up and move a small cup (or a checker introduced from week 13) from one side of the table to the other at the center of a target. The placement accuracy was measured as a function of the distance of the cup/checker to the target (Video 3). Additionally, during weeks 8-29 a modified version of the Jebsen-Taylor Hand Function Test (JHFT) ${ }^{23}$ was performed once per week to quantify functional improvement. Passive and active range of motion was also measured each week. Pinch force between the index finger and thumb during FES was measured each week using a digital pinch gauge (Baseline digital pinch gauge, Fabrication Enterprises, USA). Due to the Covid19 pandemic, functional assessments were unable to be performed during the at-home testing. 
204 During the in-laboratory portion of the study, the subject underwent weekly interviews to assess for adverse events and was also surveyed for changes in self-perceived functional independence. Changes in health status were assessed with the MOS 36-item short form health survey (SF-36). ${ }^{24}$ Perceived changes in functional independence were assessed with the Spinal Cord Independence Measure (SCIM) ${ }^{25}$ version III which ranges from 0 to 100 and higher score indicates increased independence. Detailed neurological evaluation for documentation of level and severity of SCI was conducted monthly according to the ISNCSCI. ${ }^{21}$ During home-use, the SCIM and SF-36 were administered once per week.

\section{Results}

\section{Decoder Performance}

214 Figure 2A summarizes decoding performance across all in-laboratory upper extremity sessions (open215 loop and closed loop) for weeks 9-19 for different classifier types. For offline analysis of the closed loop 216 experiments, a total of 80-240 trials were used with half of the data set used for training and the other 217 half for testing. The accuracies presented represent the average of 100 random split cross-validation 218 iterations. Mean online decoding accuracy per week was 89.0\% (median 88.75\%, range 78-93.3\%) which was not significantly different from offline performance across the 5 types of classifiers tested 220 (Kruskall-Wallis test with Tukey-Kramer adjustment for multiple comparisons, $\mathrm{p}>0.06$ ). Online decoding during weeks 9-19 remained relatively stable for upper extremity tasks across weeks as shown in Panel 2B.

The decoder trained for the at-home setting performed very well on non-ambiguous windows of data, i.e. those that did not contain mixed move/rest signals, in both open-loop and closed-loop trials (Figure 2C, D). Using the decoded probability of motor intent, the area under the receiver-operator characteristic curve (AUC) was calculated for each window of non-ambiguous data. Open-loop and closed-loop trials had similar decoding performance with AUC of 0.98 and 0.97 , and accuracy with a mean of $91.3 \%$ (median 90.8\%) and 88.3\% (median 90.3\%) respectively. During state transitions, in which windows 
contained changes in motor intent, there was an average delay of 2.3 seconds to transition to the intended motor state. These delays in time to motor intent are seen in a time series data sample (Figure 2E) where the change in the decoded state lags changes in the prompt. This delay contributes to a slightly decreased accuracy (0.69) when analyzing data across windows where motor intent changes for both open-loop $(A \cup C=0.78)$ and closed-loop $(A \cup C=0.77)$ trials.

\section{4}

235

\section{Functional improvement}

The subject showed improvement in the accuracy of placing a small cup, $60.1 \% \pm 7.8 \%$ (mean \pm std) at week 11 versus $82.8 \% \pm 4.7 \%$ at week 19 (two-tailed t-test, $p=0.03)$ or a checker $(64.5 \% \pm 7.3 \%$ at week 13 versus $88.8 \% \pm 4.8 \%$ at week 19 , two-tailed t-test, $p=0.03$ ) at the center of a target as summarized in Figure $\mathbf{3 A}$ and $3 \mathbf{B}$.

Functional improvement was quantified as the reduction in the average time taken to perform specific components of the JHFT (Figure 3C). Significant improvements were observed in lifting small objects, lifting light cans, and lifting heavy cans through orthotic-assisted tasks. Along with a trend towards improvement in writing speed (32.3s to $26.4 \mathrm{~s}$, two-tailed t-test, $p=0.15)$, clarity of the handwriting also improved throughout the course of the study (Figure 3D). Further, pinch force increased from 1lb to 3lb within 10 weeks.

\section{Clinical Assessments}

While there was no change in ISNCSCI ASIA impairment scale from a C5 motor level, there was an unexpected slight increase in the motor zone of partial preservation (defined as the myotomes below the level of injury with residual innervation) on the left from C6 to C8. Additionally, after study week 23, the subject began to be able to extend his right thumb volitionally with motor strength $2 / 5$ in the absence of the FES orthosis (Video 4). During the laboratory portion of the study, there was no change in the SCIM from a baseline score of 26 . The SF-36 indicated a $32.5 \%$ improvement in pain, a $5 \%$ increase in energy, and an $8 \%$ decrease in emotional well-being. Interestingly, during the in-home portion of the study, there was a $22.5 \%$ increase in pain (from $100 \%$ down to $77.5 \%$ in the setting of a newly diagnosed 
and treated UTI) and a one point increase in the SCIM (from 26 to 27 due to improvement in self-care (see Supplementary Methods Tables S7 and S7 for a breakdown of scores).

256

257

\section{Discussion}

Successive continuous movement of the hands were first noticed by Jasper and Penfield in 1949 to produce a blocking of the beta rhythm in the pre- and post-central hand area as measured in ECoG. ${ }^{26}$ Interestingly, the reductions in the beta power band observed in the ECoG, called event-related desynchronizations (ERDs), are also observed during imagined movements of the limb. Therefore it is not surprising that ERDs and other changes in the frequency characteristics of the EEG and ECoG have been investigated by numerous researchers as potential control signals to trigger stimulation of paralyzed muscles ${ }^{20}$ or to control the position of a cursor on a computer screen $^{18}$. A fully implanted ECoG-based $\mathrm{BCl}$ using ERDs within the ECoG signals, has been developed to allow typing in a fully locked-in patient with ALS. ${ }^{27}$ However, to our knowledge, no prior fully implanted and portable motor $\mathrm{BCl}$ has been successfully deployed in a home environment to allow volitional restoration of hand grasp.

Our results demonstrate that a fully implanted $\mathrm{BCl}$ can be safely and reliably used to decode movement intent from motor cortex allowing for volitional control of hand grasp by a patient with SCI in laboratory and home environment. In closed-loop experiments, movement intent was decoded from real-time ECoG recordings obtained from electrodes placed on the hand/arm motor cortex and the output of the classifier was used to trigger an external assistive device with a high degree of accuracy. The performance of the decoder has remained stable for the 22 months since initial device implantation with median accuracy around $90 \%$. Additionally, there was significant improvement in both the accuracy and speed of several functional hand tasks and improvements in self-perceived pain and energy scores during the initial laboratory testing period. Interestingly, there was a slight increase in the SCIM during home-use within the area of self-care as well as changes in pain perception with the laboratory and home setting. The clinical significance and stability of these perceived changes remains unclear at this time but will ultimately be crucial to understand in order to maximize the utility of $\mathrm{BCls}$ to patients with SCl. 
280 Our results show that a $\mathrm{BCl}$ that allows control of one degree of freedom (namely hand grasp) may be

281 a helpful tool for patients with paralysis to gain functional independence by allowing them to exert

282 volitional control of external devices. Although the current study did not use functional independence

283

284

285

286

287

288

289

290

291

292

293

294

295

296

297

298

299

300

301

302

303 measures aside from that captured in the SCIM, we envision that as these technologies transition out of the laboratory setting, the potential improvements in ability to perform activities of daily living will drive functional independence. Additionally, the system can be easily adapted to allow volitional control of a wide range of external devices (e.g. as a trigger for robotic assisted stepping within an exoskeleton as shown in the Supp. Methods) which may open the door for future development of assistive devices within a home environment that can be controlled with the implanted $\mathrm{BCl}$.

The main limitation of this work, the ability to decode only one degree of freedom, is driven by currently available technology. As newer devices with increased recording capabilities (increased number of channels and spatial density, and higher sampling frequency), such as the one recently developed by Benabid et al. ${ }^{19}$, become available within the US market, we anticipate that the techniques presented here-in will be generalizable to the restoration of a larger number of degrees of freedom in patients with SCI. Particularly, the development of these new devices in parallel with advanced mobile robotic exoskeleton technology may in the near future allow for the restoration of both upper and lower extremity function for patients with SCI.

\section{Support}

Supported by a private institutional grant from the Miami Project to Cure Paralysis. Device was donated by Medtronic. Medtronic provided all components of the implant including the external antenna/receiver free of charge to the University of Miami but did not provide funds directly to the institution, the researchers, or the patient. Home deployment orthosis was funded by a private donor. IC was supported in part by NIH R25NS108937-02. KD was supported in part by grant NIH T32GM112601. JHA was supported in part by NIH/NIA F32 AG064886. 


\section{Acknowledgements}

305

306

307

308

309

310

311

312

313

314

315

316

317

318

319

320

321

322

323

324

325

326

327

328

329

330

331

332

333

334

335

336

337

338

339

340

We would like to thank our subject for his continued dedication and enthusiasm throughout the duration of this study. We would like to thank Roberto Suazo for his assistance with the design of the figures and editing of videos; the dedicated staff in the operating room and intensive care unit for their post-operative care of the subject. We are thankful to $\operatorname{Dr}(\mathrm{s})$ Roberto C. Heros, Barth A. Green, Alexander Koenig, and George M. Ibrahim for their comments on initial drafts of the manuscript.

\section{Data Sharing}

Individual participant data that underlies the results reported in this article after de-identification, study protocol, statistical analysis plan, and analytic code will be made available upon request to researches who provide a methodologically sound proposal. Requests should be made to icajigas@med.miami.edu.

\section{References}

1. Anderson, K.D. Consideration of user priorities when developing neural prosthetics. J Neural Eng 6, 055003 (2009).

2. Aravamudhan, S. \& Bellamkonda, R.V. Toward a convergence of regenerative medicine, rehabilitation, and neuroprosthetics. J Neurotrauma 28, 2329-2347 (2011).

3. Armour, B.S., Courtney-Long, E.A., Fox, M.H., Fredine, H. \& Cahill, A. Prevalence and Causes of ParalysisUnited States, 2013. Am J Public Health 106, 1855-1857 (2016).

4. Nicolelis, M.A. Brain-machine interfaces to restore motor function and probe neural circuits. Nat Rev Neurosci 4, 417-422 (2003).

5. Wolpaw, J.R., et al. Brain-computer interface technology: a review of the first international meeting. IEEE Trans Rehabil Eng 8, 164-173 (2000).

6. Wolpaw, J.R., Birbaumer, N., McFarland, D.J., Pfurtscheller, G. \& Vaughan, T.M. Brain-computer interfaces for communication and control. Clin Neurophysiol 113, 767-791 (2002).

7. Ajiboye, A.B., et al. Restoration of reaching and grasping movements through brain-controlled muscle stimulation in a person with tetraplegia: a proof-of-concept demonstration. Lancet 389, 1821-1830 (2017).

8. Chapin, J.K., Moxon, K.A., Markowitz, R.S. \& Nicolelis, M.A. Real-time control of a robot arm using simultaneously recorded neurons in the motor cortex. Nat Neurosci 2, 664-670 (1999).

9. Collinger, J.L., et al. High-performance neuroprosthetic control by an individual with tetraplegia. Lancet 381, 557-564 (2013).

10. Hochberg, L.R., et al. Reach and grasp by people with tetraplegia using a neurally controlled robotic arm. Nature 485, 372-375 (2012).

11. Hochberg, L.R., et al. Neuronal ensemble control of prosthetic devices by a human with tetraplegia. Nature 442, 164-171 (2006).

12. Velliste, M., Perel, S., Spalding, M.C., Whitford, A.S. \& Schwartz, A.B. Cortical control of a prosthetic arm for self-feeding. Nature 453, 1098-1101 (2008). 
13. Bouton, C.E., et al. Restoring cortical control of functional movement in a human with quadriplegia. Nature 533, 247-250 (2016).

14. Gunasekera, B., Saxena, T., Bellamkonda, R. \& Karumbaiah, L. Intracortical recording interfaces: current challenges to chronic recording function. ACS Chem Neurosci 6, 68-83 (2015).

15. Heasman, J.M., et al. Control of a hand grasp neuroprosthesis using an electroencephalogram-triggered switch: demonstration of improvements in performance using wavepacket analysis. Med Biol Eng Comput 40, 588-593 (2002).

16. Meng, J., et al. A Study of the Effects of Electrode Number and Decoding Algorithm on Online EEG-Based BCI Behavioral Performance. Front Neurosci 12, 227 (2018).

17. Wang, W., et al. An electrocorticographic brain interface in an individual with tetraplegia. PLoS One 8, e55344 (2013).

18. Wolpaw, J.R. \& McFarland, D.J. Control of a two-dimensional movement signal by a noninvasive braincomputer interface in humans. Proc Natl Acad Sci U S A 101, 17849-17854 (2004).

19. Benabid, A.L., et al. An exoskeleton controlled by an epidural wireless brain-machine interface in a tetraplegic patient: a proof-of-concept demonstration. Lancet Neurol 18, 1112-1122 (2019).

20. Gant, K., et al. EEG-controlled functional electrical stimulation for hand opening and closing in chronic complete cervical spinal cord injury. Biomedical Physics \& Engineering Express 4, 065005 (2018).

21. Kirshblum, S.C., et al. International standards for neurological classification of spinal cord injury (revised 2011). J Spinal Cord Med 34, 535-546 (2011).

22. Babadi, B. \& Brown, E.N. A review of multitaper spectral analysis. IEEE Trans Biomed Eng 61, 1555-1564 (2014).

23. Jebsen, R.H., Taylor, N., Trieschmann, R.B., Trotter, M.J. \& Howard, L.A. An objective and standardized test of hand function. Arch Phys Med Rehabil 50, 311-319 (1969).

24. Ware, J.E., Jr. \& Sherbourne, C.D. The MOS 36-item short-form health survey (SF-36). I. Conceptual framework and item selection. Med Care 30, 473-483 (1992).

25. Itzkovich, M., et al. The Spinal Cord Independence Measure (SCIM) version III: reliability and validity in a multi-center international study. Disabil Rehabil 29, 1926-1933 (2007).

26. Jasper, H. \& Penfield, W. Electrocorticograms in man: Effect of voluntary movement upon the electrical activity of the precentral gyrus. Archiv für Psychiatrie und Nervenkrankheiten 183, 163-174 (1949).

27. Vansteensel, M.J., et al. Fully Implanted Brain-Computer Interface in a Locked-In Patient with ALS. N Engl J Med 375, 2060-2066 (2016).

Figure 1 Pre-operative Imaging used for electrode placement, laboratory and home system setups, and illustration of electrocorticographic (ECOG) event related desynchronizations. Panel A show preoperative sagittal MRI (top) showing post-traumatic cyst centered at C4. Stereotactic navigation was used to plan a small craniotomy over the region of increased fMRI signal during imagined right hand movements which coincided with 
the hand/arm area of the precentral gyrus on the left hemisphere. Panel B shows relative location of electrodes on brain surface and configuration of data channels with respect to surface electrode contacts. Panel C shows the upper extremity laboratory setup. Real-time ECoG recordings from hand motor cortex are obtained via an antenna placed over the implanted transmitter. The antenna is connected to a receiver that then connects to laptop computer. The subject is prompted to think about resting or moving his right hand during a computer task and the signals recorded from the channels shown in Panel B are processed to build classifiers that can be used to classify when the subject is thinking about move or rest. When a move state is correctly decoded, functional electrical stimulation of the right hand is applied to the subject using a FES orthosis. Panel D shows the portable $\mathrm{BCl}$ system setup. Note that the FES orthosis has been replaced by a motorized hand orthosis. Panel $\mathrm{E}$ center shows the average spectrogram for the continuous time channels ( 1 and 3 ) over all upper extremity task along with corresponding average power as well as the average power spectral density (PSD) for move and rest states for each channel. All PSDs have confidence intervals calculated by the standard error of the mean. As can be clearly seen by the central spectrogram, motor imagery causes a decrease in the power in the beta and low gamma frequencies of the ECoG.

Figure 2 Upper extremity decoding performance. Panel A shows the accuracy of different types of classifiers to decode rest/move states during the hand task in the laboratory. Best online and off-line in-laboratory performance was seen with bagged-tree classifier - 89.0\% (median 88.75\%, range 78-93.3\%). Panel B shows that the decoding accuracy remained relatively stables over the 10 weeks of upper extremity tasks. Panel $\mathrm{C}$ shows the performance of the at-home decoder under open-loop and closed-loop settings. Panel D shows the distribution of at-home decoding accuracies under open-loop $(\mathrm{N}=13)$ and closed-loop $(\mathrm{N}=12)$ settings. Panel $E$ shows a sample at-home time series during an accuracy assessment demonstrating the movement state being displayed to the subject, the decoder movement state probability, and the decoded state.

Figure 3 Functional task structure and performance. Panel A shows the setup for the checker and cup task.

406 The subject was instructed to try to place the corresponding object at the center of the target $(n=20)$ and this task was repeated 3 times during a study week visit. Panel B shows significant improvement in accuracy from week 11 to study week 19 . Panel $\mathrm{C}$ shows comparison of times between study week 9 and 19 for different components 
409 of the JHFT. Each JHFT task was repeated a total of 5 times per session. Bar height corresponds to mean times $410 \pm$ std; $p$-values computed with two-tailed t-test. Panel $D$ shows the best handwriting sample from each week from 411 week 10-29 along with average time to write each of the words. Each word was written a total of 5 times per week. 
A

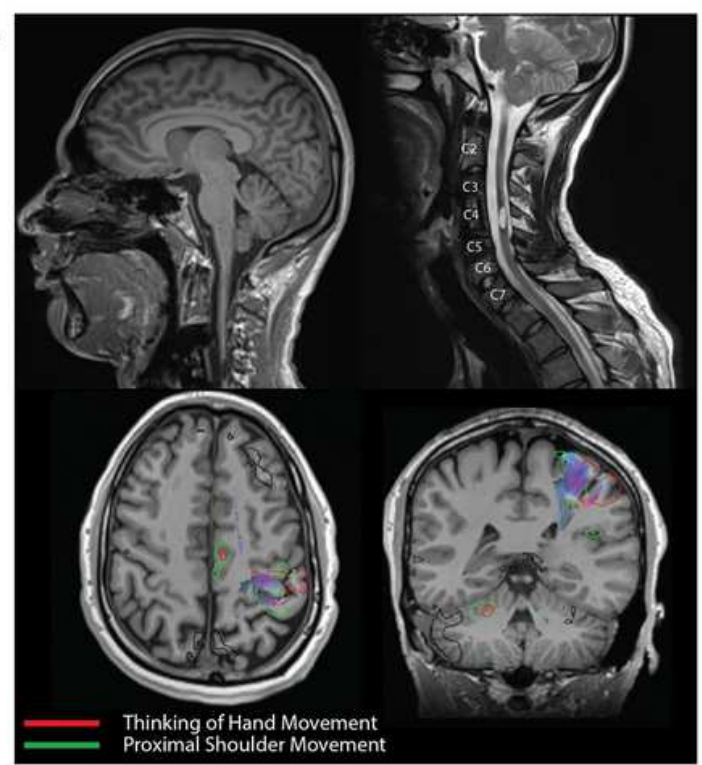

D

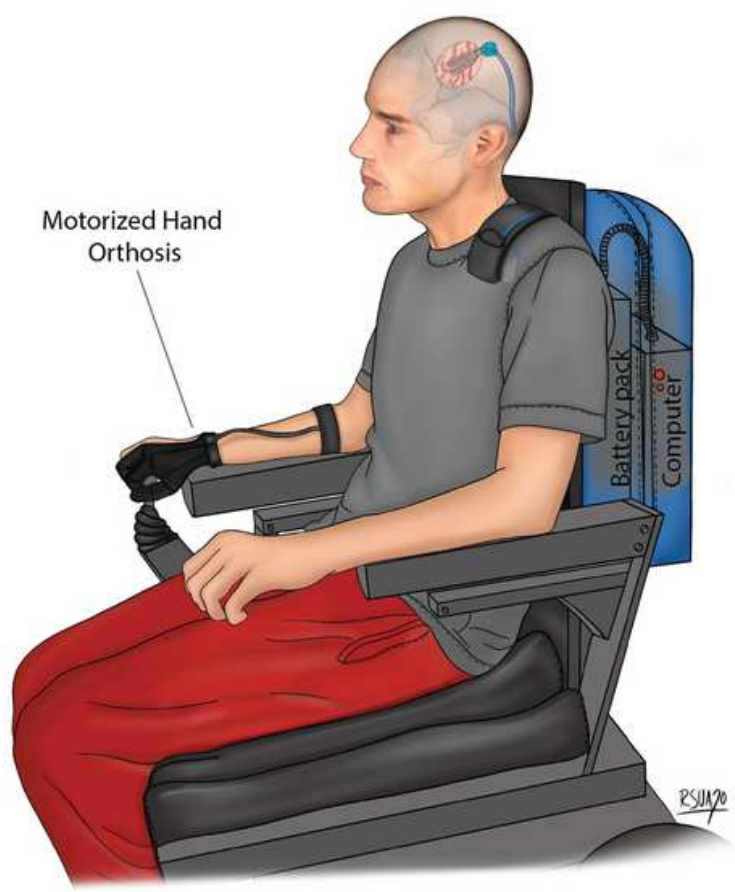

B

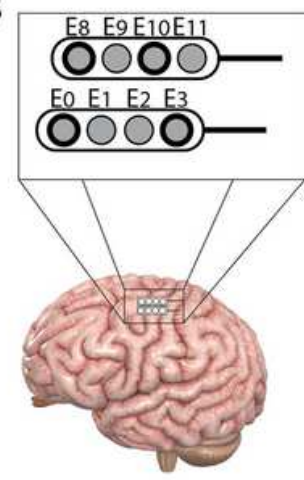

Time Channels

Channel 1: E0-E3

Channel 3: E8 - E10

Power Channels

Channel 2: E1 - E2

Channel 4: E9 - E11

E
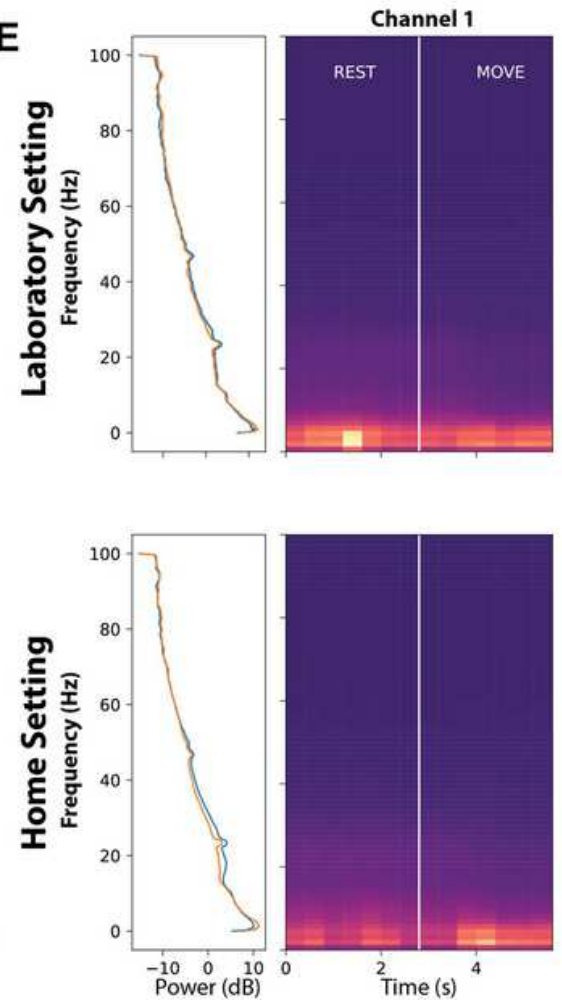

C
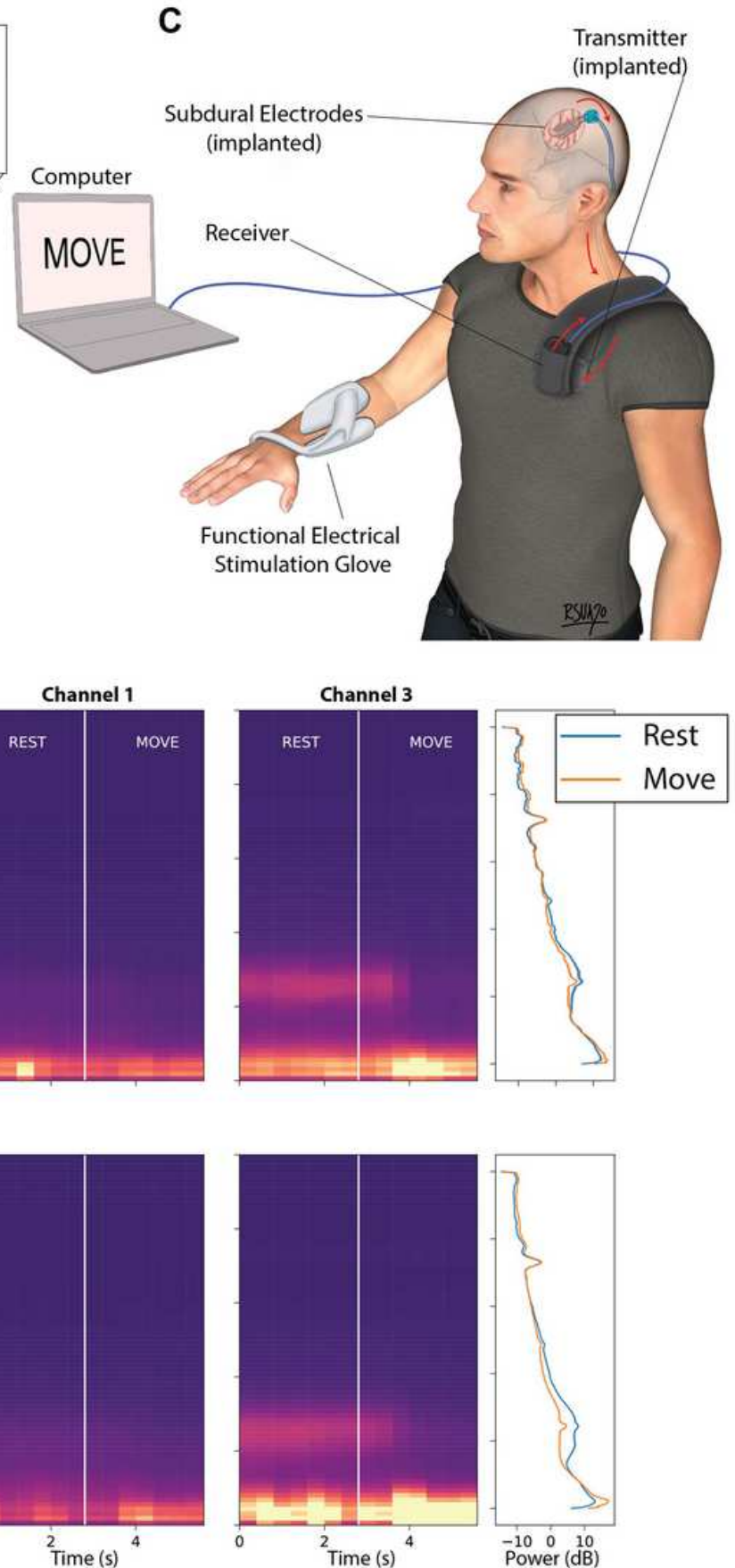

\section{Figure 1}

Pre-operative Imaging used for electrode placement, laboratory and home system setups, and illustration of electrocorticographic (ECoG) event related desynchronizations. Panel A show preoperative sagittal MRI (top) showing post-traumatic cyst centered at C4. Stereotactic navigation was used to plan a small craniotomy over the region of increased fMRI signal during imagined right hand movements which coincided with the hand/arm area of the precentral gyrus on the left hemisphere. Panel B shows relative location of electrodes on brain surface and configuration of data channels with respect to surface electrode contacts. Panel C shows the upper extremity laboratory setup. Real-time ECoG recordings from 
hand motor cortex are obtained via an antenna placed over the implanted transmitter. The antenna is connected to a receiver that then connects to laptop computer. The subject is prompted to think about resting or moving his right hand during a computer task and the signals recorded from the channels shown in Panel B are processed to build classifiers that can be used to classify when the subject is thinking about move or rest. When a move state is correctly decoded, functional electrical stimulation of the right hand is applied to the subject using a FES orthosis. Panel D shows the portable $\mathrm{BCl}$ system setup. Note that the FES orthosis has been replaced by a motorized hand orthosis. Panel E center shows the average spectrogram for the continuous time channels ( 1 and 3 ) over all upper extremity task along with corresponding average power as well as the average power spectral density (PSD) for move and rest states for each channel. All PSDs have confidence intervals calculated by the standard error of the mean. As can be clearly seen by the central spectrogram, motor imagery causes a decrease in the power in the beta and low gamma frequencies of the ECoG. 
A

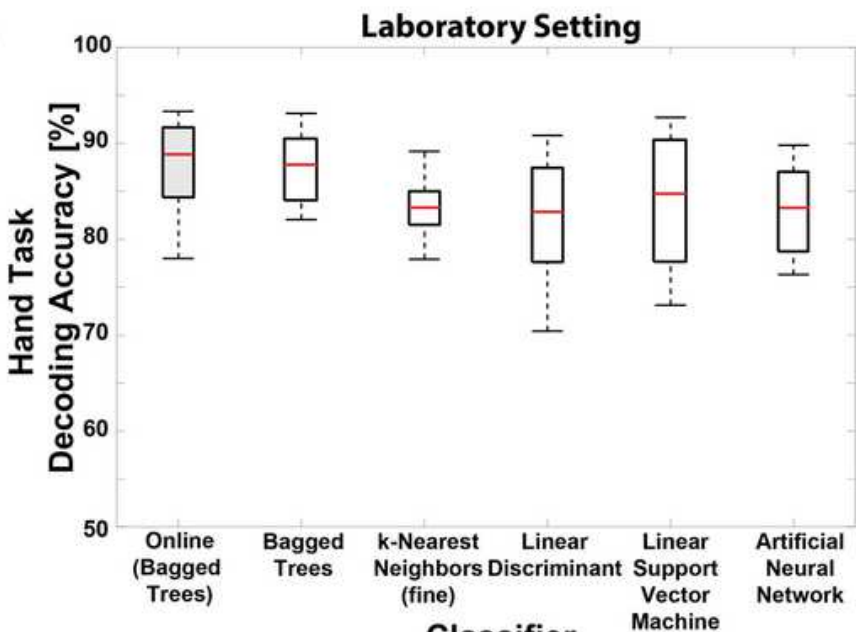

Classifier

C

$E$

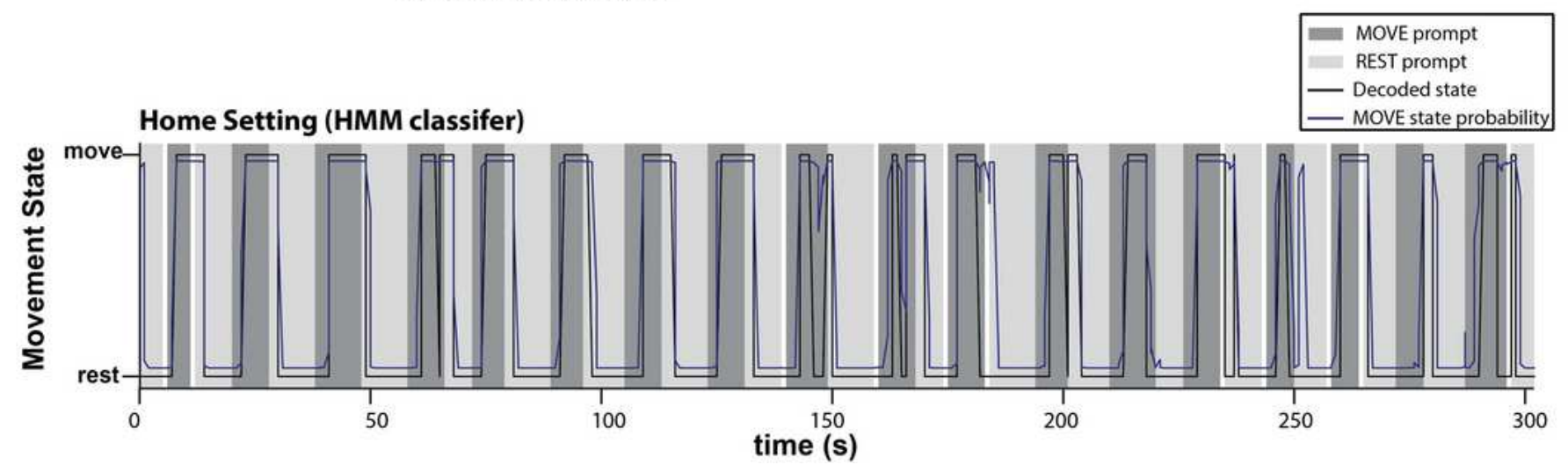

Figure 2

Upper extremity decoding performance. Panel A shows the accuracy of different types of classifiers to decode rest/move states during the hand task in the laboratory. Best online and off-line in-laboratory performance was seen with bagged-tree classifier - 89.0\% (median 88.75\%, range 78-93.3\%). Panel B shows that the decoding accuracy remained relatively stables over the 10 weeks of upper extremity tasks. Panel $\mathrm{C}$ shows the performance of the at-home decoder under open-loop and closed-loop settings. Panel 
D shows the distribution of at-home decoding accuracies under open-loop ( $N=13)$ and closed-loop $(\mathrm{N}=12)$ settings. Panel E shows a sample at-home time series during an accuracy assessment demonstrating the movement state being displayed to the subject, the decoder movement state probability, and the decoded state.

A

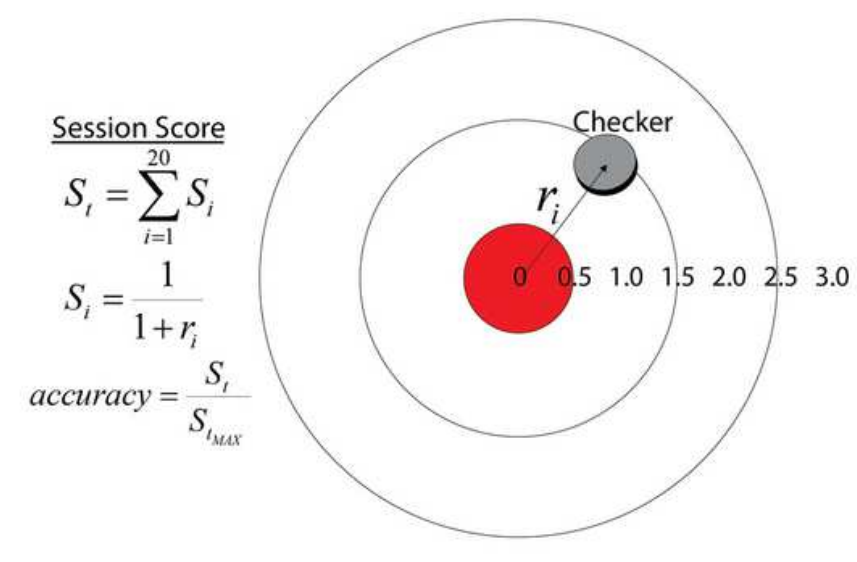

C

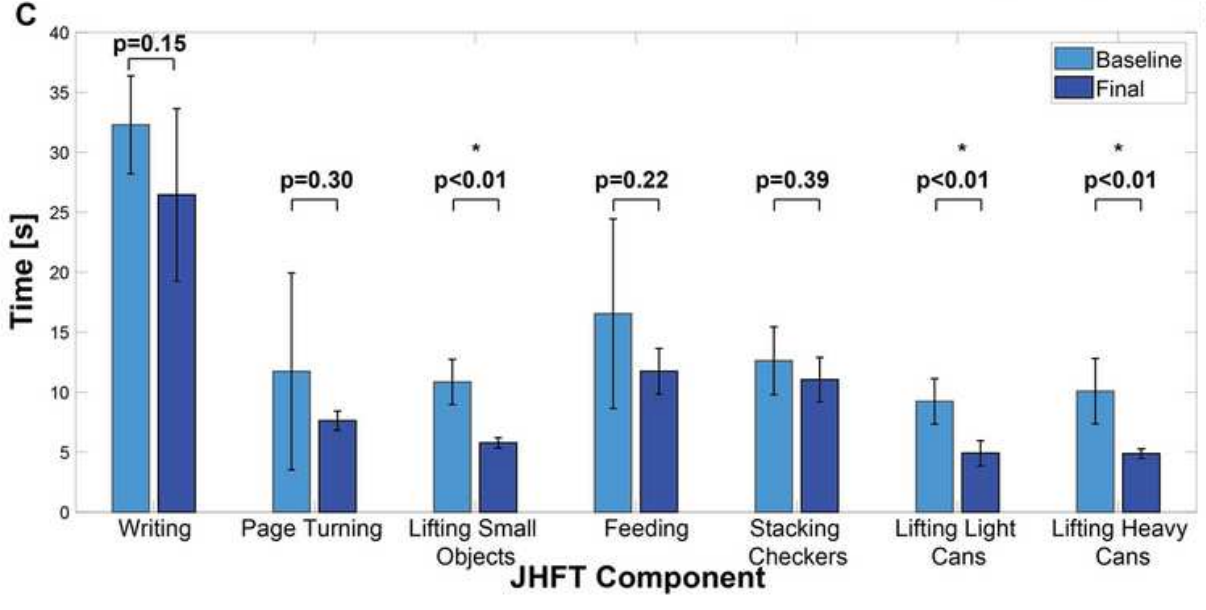

B

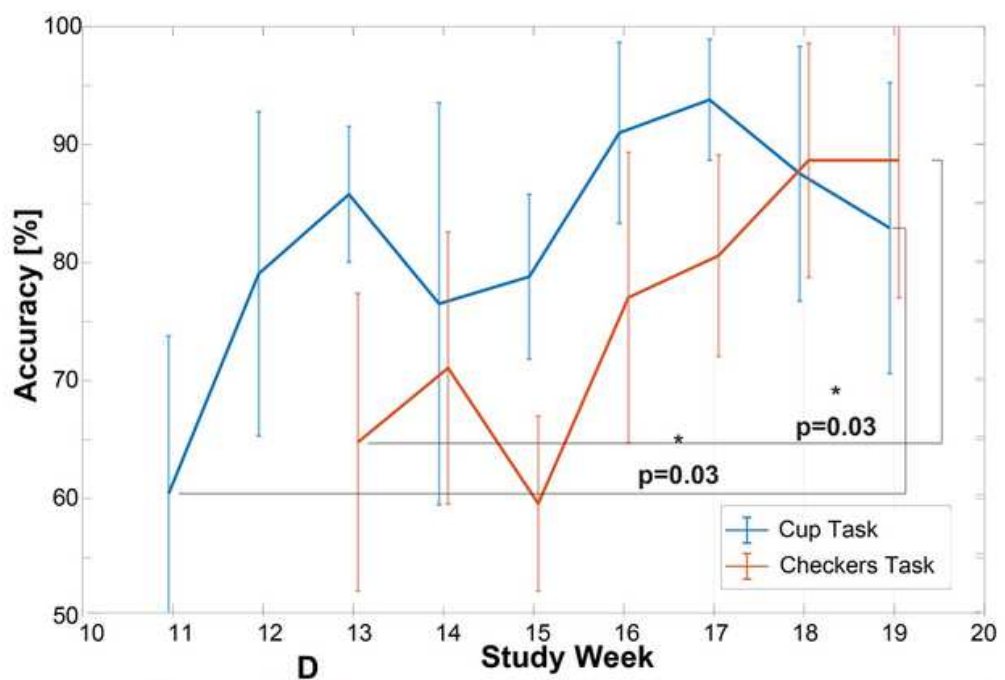

Figure 3

Functional task structure and performance. Panel A shows the setup for the checker and cup task. The subject was instructed to try to place the corresponding object at the center of the target $(n=20)$ and this task was repeated 3 times during a study week visit. Panel B shows significant improvement in accuracy from week 11 to study week 19. Panel C shows comparison of times between study week 9 and 19 for different components of the JHFT. Each JHFT task was repeated a total of 5 times per session. Bar height corresponds to mean times \pm std; $p$-values computed with two-tailed t-test. Panel $D$ shows the best handwriting sample from each week from week 10-29 along with average time to write each of the words. Each word was written a total of 5 times per week.

\section{Supplementary Files}

This is a list of supplementary files associated with this preprint. Click to download. 
- BMISupplementaryMethods1052020.pdf

- Video1.mp4

- Video2conv.mp4

- Video3.mp4

- Video4.mp4

- VideoS1.mp4 